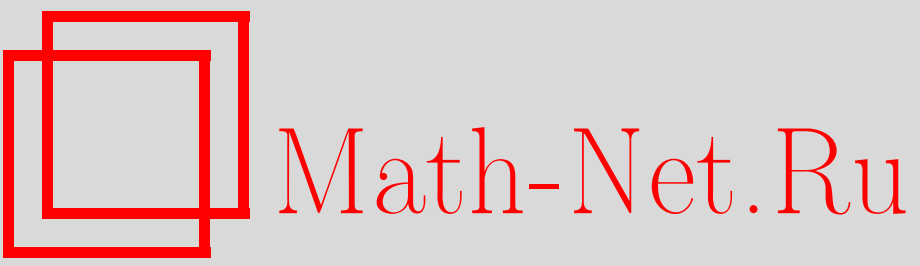

В. И. Паньженский, О. П. Сурина, Субримановы геодезические на многомерной группе Гейзенберга, Итоги науки и техн. Сер. Соврем. мат. и ее прил. Темат. обз., 2020, том 180, 74-84

DOI: https://doi.org/10.36535/0233-6723-2020-180-74-84

Использование Общероссийского математического портала Math-Net.Ru подразумевает, что вы прочитали и согласны с пользовательским соглашением

http: //www. mathnet.ru/rus/agreement

Параметры загрузки:

IP : 34.229 .108 .108

26 апреля 2023 г., 14:25:57 


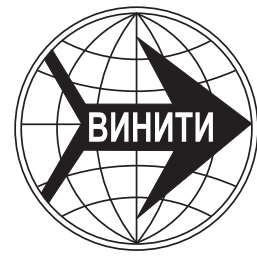

ИТОГИ НАУКИ И ТЕХНИКИ.

Современная математика и ее приложения.

Тематические обзоры.

Том 180 (2020). С. $74-84$

DOI: $10.36535 / 0233-6723-2020-180-74-84$

УДК 514.763

\title{
СУБРИМАНОВЫ ГЕОДЕЗИЧЕСКИЕ НА МНОГОМЕРНОЙ ГРУППЕ ГЕЙЗЕНБЕРГА
}

\author{
() 2020 г. В. И. ПАНЬЖЕНСКИЙ, О. П. СУРИНА
}

\begin{abstract}
АннотАция. Изучается строение геодезических усеченной связности на многомерной группе Гейзенберга, наделенной левоинвариантной субримановой структурой. Установлено, что субримановыми геодезическими являются параболы, ортогональные проекции которых на соответствующие контактные плоскости являются прямыми линиями. Кроме таких парабол, геодезическими являются также некоторые прямые, лежащие в контактных плоскостях.
\end{abstract}

Ключевые слова: группа Гейзенберга, субриманова структура, левоинвариантная контактная метрическая структура, геодезическая.

\section{SUB-RIEMANNIAN GEODESICS ON THE MULTIDIMENSIONAL HEISENBERG GROUP}

\author{
(c) 2020 V. I. PANZHENSKII, O. P. SURINA
}

\begin{abstract}
In this paper, we study the structure of truncated connections on the multidimensional Heisenberg group endowed with a left-invariant sub-Riemannian structure. We find that subRiemannian geodesics are parabolas whose orthogonal projections onto the corresponding contact planes are straight lines. In addition to such parabolas, some straight lines lying in contact planes are also geodesics.
\end{abstract}

Keywords and phrases: Heisenberg group, sub-Riemannian structure, left-invariant contact metric structure, geodesic.

AMS Subject Classification: 53B21

1. Введение. Группа Гейзенберга - это трехмерная связная односвязная нильпотентная группа Ли $G$, состоящая из всех вещественных верхнетреугольных матриц с единицами на главной диагонали. На этой группе имеется левоинвариантная контактная дифференциальная 1-форма $\eta$ и полная риманова метрика $g$, которые задают на $G$ контактную метрическую структуру. Вполне неголономное контактное распределение $\operatorname{ker} \eta$ вместе с ограничением метрики $g$ на это распределение определяет на $G$ субриманову структуру. Среди трехмерных групп Ли группа Гейзенберга является одним из наиболее известных и простых примеров в исследованиях по субримановой геометрии, теории динамических систем и теории оптимального управления (см. $[1,2,4,8,9]$ ). В подобных исследованиях часто геодезическими называют экстремали вариационной задачи на минимум функционала длины кривой. В неголономной механике считается, что механическая система с лагранжианом $L$ и неголономным распределением на конфигурационном пространстве движется по траектории, которая, вообще говоря, не является решением вариационной задачи на минимум. В [3] доказано, что траектории движения такой механической системы с квадратичным лагранжианом являются геодезическими усеченной связности. 
В настоящей работе изучается строение геодезических усеченной связности на многомерной группе Гейзенберга, наделенной левоинвариантной субримановой структурой. Установлено, что субримановыми геодезическими являются параболы, ортогональные проекции которых на соответствующие контактные плоскости являются прямыми линиями. Кроме таких парабол геодезическими являются также некоторые прямые, лежащие в контактных плоскостях.

В разделе 2 приведены основные определения. Показано, как с помощью левых сдвигов на многомерной группе Гейзенберга можно получить левоинвариантные контактную форму и риманову метрику и как они порождают контактную метрическую структуру, которая является сасакиевой. Доказано, что для многомерной группы Гейзенберга имеет место утверждение о существовании единственности контактной метрической связности с кососимметрическим кручением, установленное в [7] для трехмерной группы Гейзенберга. При этом, в отличие от трехмерного случая, мы дополнительно требуем инвариантность связности относительно автоморфизмов сасакиевой структуры. Доказано также, что обнаруженная связность является контактной метрической связностью для любой $k$-контактной метрической структуры и, следовательно, для любой сасакиевой структуры.

В разделе 3 вычислены компоненты связности Леви-Чивиты и контактной метрической связности в ортонормированном репере, адаптированном к контактной структуре. Проектирование этой связности на контактное распределение приводит к одной и той же усеченной связности. Выписывая дифференциальные уравнения субримановых геодезических, т.е. геодезических усеченной связности, касательные векторы которых лежат в контактном распределении, и интегрируя эти уравнения, находим общее решение, что позволяет выяснить строение геодезических.

В разделе 4 дана оценка секционной кривизны, а именно, доказано, что секционная кривизна группы Гейзенберга с контактной метрической связностью принадлежит числовому отрезку $[-1 ; 0]$.

2. Контактная метрическая связность. Многомерная группа Гейзенберга - это нильпотентная $m$-мерная $(m=2 n+1)$ группой Ли $((n+2) \times(n+2))$-матриц вида

$$
G=\left(\begin{array}{ccc}
1 & y & z \\
\theta^{t} & E_{n} & x^{t} \\
0 & \theta & 1
\end{array}\right)
$$

(см. [11]), где $x^{t}=\left(x^{1}, \ldots, x^{n}\right)^{t}, y=\left(x^{n+1}, \ldots, x^{2 n}\right), \theta=(0, \ldots, 0) \in \mathbb{R}^{n}, z=x^{m} \in \mathbb{R}, E_{n}$ - единичная $(n \times n)$-матрица, $\mathbb{R}$ - поле действительных чисел. Левые сдвиги на группе $G$ определяются следующими формулами:

$$
\begin{aligned}
\bar{x}^{i} & =x^{i}+c^{i}, \quad i=\overline{1,2 n} ; \\
\bar{x}^{m} & =x^{m}+c^{n+1} x^{1}+\ldots+c^{2 n} x^{n}+c^{m} .
\end{aligned}
$$

Дифференцируя по параметрам и вычисляя полученные производные в единице группы, находим базисные левоинвариантные векторные поля:

$$
X_{\alpha}=\partial_{\alpha}, \quad X_{n+\alpha}=\partial_{n+\alpha}+x_{\alpha} \partial_{m}, \quad X_{m}=\partial_{m}, \quad x_{\alpha}=x^{\alpha} ; \alpha=\overline{1, n} ; \partial_{k}=\frac{\partial}{\partial x^{k}} .
$$

Левоинвариантную риманову метрику на группе $G$ можно получить следующим образом. В касательном пространстве единицы группы $T_{e} G$ возьмем евклидову метрику $d s^{2}=d x^{1^{2}}+\ldots+d x^{m 2}$ и «сдвинем» ее в произвольную точку $\bar{x}$. Так как

$$
d x^{i}=d \bar{x}^{i}, \quad d x^{m}=d \bar{x}^{m}-c^{n+1} d \bar{x}^{1}-\ldots-c^{2 n} d \bar{x}^{n}, \quad c^{k}=\bar{x}^{k},
$$

то

$$
d \bar{s}^{2}=d \bar{x}^{12}+\ldots+d \bar{x}^{2 n^{2}}+\left(d \bar{x}^{m}-\bar{x}^{n+1} d \bar{x}^{1}-\ldots-\bar{x}^{2 n} d \bar{x}^{n}\right)^{2} .
$$

Аналогично можно получить левоинвариантную дифференциальную 1-форму, взяв в касательном пространстве единицы группы форму $d x^{m}$ и сдвинув ее в точку $\bar{x}$. В результате получим

$$
d \bar{x}^{m}-\bar{x}^{n+1} d \bar{x}^{1}-\ldots-\bar{x}^{2 n} d \bar{x}^{n} .
$$


Таким образом, левоинвариантные форма $\eta$ и риманова метрика $g$ имеют вид

$$
\begin{gathered}
\eta=d x^{m}-x^{n+1} d x^{1}-\ldots-x^{2 n} d x^{n} \\
d s^{2}=d x^{1^{2}}+\ldots+d x^{2 n^{2}}+\left(d x^{m}-x^{n+1} d x^{1}-\ldots-x^{2 n} d x^{n}\right)^{2}
\end{gathered}
$$

и определяют на группе Гейзенберга контактную метрическую структуру $(\eta, \xi, \Phi, g)$. Действительно, так как $\eta \wedge(d n)^{n} \neq 0$ ( $\wedge$ - внешнее произведение, $d-$ внешний дифференциал), то форма $\eta$ является контактной и определяет контактное $2 n$-мерное распределение $\mathfrak{L}=\operatorname{ker} \eta$. Контактное распределение $\mathfrak{L}$ называется первым фундаментальным распределением, или горизонтальным распределением, а 1-мерное распределение $\mathfrak{M}=\operatorname{ker} d \eta$ - вторым фундаментальным распределением, или вертикальным распределением. Характеристическое векторное поле $\xi$ и структурный эндоморфизм $\Phi$ однозначно определяются условиями

$$
\eta(Y)=g(\xi, Y), \quad d \eta(X, Y)=g(\Phi X, Y) .
$$

Теперь нетрудно убедиться, что выполняются условия

$$
\begin{gathered}
\eta(\xi)=1, \\
\eta \circ \Phi=0, \\
\Phi(\xi)=0, \\
\Phi^{2}=-\mathrm{id}+\eta \otimes \xi, \\
g(\Phi X, \Phi Y)=g(X, Y)-\eta(X) \eta(Y)
\end{gathered}
$$

и, следовательно, четверка тензорных полей $(\eta, \xi, \Phi, g)$ определяет на группе Гейзенберга контактную метрическую структуру. Кроме того, нетрудно убедиться, что $N_{\Phi}+2 d \eta \otimes \xi=0$, где $N_{\Phi}$ - тензор Нейенхейса структурного эндоморфизма, т.е. контактная метрическая структура, является нормальной и, следовательно, сасакиевой.

Линейная связность $\widetilde{\nabla}$ называется контактной метрической связностью, если в этой связности контактная форма $\eta$ и метрический тензор $g$ ковариантно постоянны: $\widetilde{\nabla} \eta=0, \widetilde{\nabla} g=0$. При параллельном перенесении векторов в этой связности сохраняются длины векторов и углы между векторами (связность метрическая: $\widetilde{\nabla} g=0$ ) и контактное распределение $\mathfrak{L}(\widetilde{\nabla} \eta=0)$, а, следовательно, и распределение $\mathfrak{M}$. Из соотношения $\widetilde{\nabla} \eta=0$ следует, что связность $\widetilde{\nabla}$ необходимо имеет кручение $\widetilde{S}$, а из $\widetilde{\nabla} g=0$ следует, что ковариантный тензор деформации $T$ связности Леви-Чивиты $\nabla$ кососимметричен по последним двум аргументам. Если тензор $T$ кососимметричен по всем своим аргументам, то имеем связность $\widetilde{\nabla}$ с кососимметрическим кручением. В этом случае $T=\frac{1}{2} S$ и

$$
\widetilde{\Gamma}_{i j}^{k}=\Gamma_{i j}^{k}+\frac{1}{2} S_{i j}^{k}
$$

где $\widetilde{\Gamma}_{i j}^{k}$ - коэффициенты связности $\widetilde{\nabla}, \Gamma_{i j}^{k}$ - коэффициенты связности Леви-Чивиты $\nabla, S_{i j}^{k}-$ компоненты тензора кручения $\widetilde{S}$, а $S_{i j k}=S_{i j}^{p} g_{k p}$ кососимметричны по всем индексам и, следовательно, определяют дифференциальную 3 -форму $S$ - форму кручения; $i, j, k, \ldots=\overline{1, m}$.

Теорема 1. На группе Гейзенберга существует единственная контактная метрическая связность с кососимметрическим кручением, инвариантная относительно группь автоморфизмов сасакиевой структуры. Эта связность определяется следующей формулой

$$
g\left(\widetilde{\nabla}_{X} Y, Z\right)=g\left(\nabla_{X} Y, Z\right)+\frac{1}{2} d \eta(X, Y) \wedge \eta(Z),
$$

где $X, Y, Z$ - векторные поля на $G$.

Доказательство. Заметим, что компоненты контактной формы $\eta$ и метрического тензора $g$ зависят только от переменных $x^{n+\alpha}$, поэтому $\partial_{\alpha} \eta_{i}=0, \partial_{m} \eta_{i}=0, \partial_{\alpha} g_{i j}=0, \partial_{m} g_{i j}=0$. Кроме того, $\xi=\partial_{m}$ и, следовательно, $g^{i p} \eta_{p}=\xi^{i}=\delta_{m}^{i}$, где $\delta_{m}^{i}$ - символ Кронекера. 
Теперь мы можем найти условия на компоненты формы кручения, которые следуют из ковариантного постоянства контактной формы

$$
\widetilde{\nabla}_{i} \eta_{j}=\partial_{i} \eta_{j}-\frac{1}{2} g^{k p}\left(\partial_{i} g_{p j}+\partial_{j} g_{i p}-\partial_{p} g_{i j}\right) \eta_{k}-\frac{1}{2} S_{i j p} g^{p k} \eta_{k}=0 .
$$

С учетом вышесказанных замечаний эти уравнения примут вид

$$
\partial_{i} \eta_{j}-\frac{1}{2}\left(\partial_{i} g_{m j}+\partial_{j} g_{i m}\right)-\frac{1}{2} S_{i j m}=0 .
$$

Придавая индексам $i, j$ различные значения, получим следующие условия на компоненты формы кручения

$$
S_{\alpha \beta m}=0, \quad S_{n+\alpha n+\beta m}=0, \quad S_{\alpha n+\beta m}=\delta_{\alpha \beta}, \quad S_{n+\alpha \beta m}=-\delta_{\alpha \beta} .
$$

Таким образом, из условия ковариантного постоянства контактной формы следует, что

$$
S_{\alpha n+\alpha m}=S_{n+\alpha m \alpha}=S_{n \alpha n+\alpha}=-S_{n+\alpha \alpha m}=-S_{m n+\alpha \alpha}=-S_{\alpha m n+\alpha}=1 .
$$

Остальные компоненты, содержащие индекс $m$, равны нулю. На компоненты $S_{i j k}$, не содержащие индекс $m$, ковариантная постоянность формы $\eta$ не накладывает условий.

Потребуем инвариантность связности $\widetilde{\nabla}$ относительно группы автоморфизмов сасакиевой структуры. Так как связность Леви-Чивиты $\nabla$ метрики $g$ инвариантна относительно изометрий, то она инвариантна и относительно автоморфизмов. Поэтому связность $\widetilde{\nabla}$ инвариантна тогда и только тогда, когда инвариантна форма кручения. Согласно известной теореме Танно (см. [12]), левоинвариантная сасакиева структура обладает группой Ли автоморфизмов максимальной размерности $(n+1)^{2}$. Пусть $\varphi_{t}=\exp t X-$ однопараметрическая подгруппа автоморфизмов, порожденная векторным полем $X$. Тогда производная Ли вдоль $X$ от контактной формы $\eta$ и метрического тензора $g$ равны нулю: $L_{X} \eta=0, L_{X} g=0$, т.е.

$$
\begin{gathered}
X^{p} \partial_{p} \eta_{i}+\partial_{i} X^{p} \eta_{p}=0, \\
X^{p} \partial_{p} g_{i j}+\partial_{i} X^{p} g_{p j}+\partial_{j} X^{p} g_{i p}=0 .
\end{gathered}
$$

Интегрируя эти уравнения, находим общее решение, а затем и операторы группы - базисные векторные поля ее алгебры.

$$
\begin{gathered}
X_{\alpha}=\partial_{\alpha}, \quad X_{n+\alpha}=\partial_{n+\alpha}+x^{\alpha} \partial_{m}, \quad X_{m}=\partial_{m}, \\
X_{\alpha}^{\beta}=x^{\beta} \partial_{\alpha}-x^{\alpha} \partial_{\beta}+x^{n+\beta} \partial_{n+\alpha}-x^{n+\alpha} \partial_{n+\beta}, \\
X_{n+\beta}^{\beta}=x^{n+\beta} \partial_{\beta}-x^{\beta} \partial_{n+\beta}+\frac{1}{2}\left(\left(x^{n+\beta}\right)^{2}-\left(x^{\beta}\right)^{2}\right) \partial_{m}, \\
X_{n+\alpha}^{\beta}=x^{n+\beta} \partial_{\alpha}-x^{\alpha} \partial_{n+\beta}+x^{n+\alpha} \partial_{\beta}-x^{\beta} \partial_{n+\alpha}+\left(x^{n+\alpha} x^{n+\beta}-x^{\alpha} x^{\beta}\right) \partial_{m},
\end{gathered}
$$

$\alpha<\beta, \alpha, \beta=\overline{1, n}$. Заметим, что первые $2 n+1$ векторных полей являются левоинвариантными и определяют сдвиги на группе Гейзенберга, остальные векторные поля - операторы вращений.

Уравнения инвариантности формы кручения имеют вид $\left(L_{X} S=0\right)$ :

$$
X^{p} \partial_{p} S_{i j k}+\partial_{i} X^{p} S_{p j k}+\partial_{j} X^{p} S_{i p k}+\partial_{k} X^{p} S_{i j p}=0 .
$$

Эти уравнения должны обращаться в тождества для каждого из $(n+1)^{2}$ операторов группы. Найдем условия, которые накладывают на $S_{i j k}$ операторы сдвигов. Для $X_{n+\alpha}=\partial_{n+\alpha}+x^{\alpha} \partial_{m}$ имеем

$$
\partial_{n+\alpha} S_{i j k}+\delta_{i \alpha} S_{m j k}+\delta_{j \alpha} S_{i m k}+\delta_{k \alpha} S_{i j m}=0
$$

откуда следует, что

$$
\begin{gathered}
S_{\alpha, \beta, n+\alpha}=x^{n+\beta}+c^{n+\beta}, \quad S_{\alpha, \beta, n+\beta}=-\left(x^{n+\alpha}+c^{n+\alpha}\right), \\
S_{\alpha, n+\alpha, n+\beta}=c^{\beta}, \quad S_{\beta, n+\alpha, n+\beta}=c^{\alpha}
\end{gathered}
$$

$\alpha<\beta, c^{n+\beta}, c^{n+\alpha}, c^{\beta}, c^{\alpha}=$ const. 
Далее исследуем уравнения инвариантности для операторов вращения. Для $X_{\alpha}^{\beta}$ имеем следующие уравнения:

$$
\begin{aligned}
& x^{n+\beta} \partial_{n+\alpha} S_{i j k}-x^{n+\alpha} \partial_{n+\beta} S_{i j k}+\delta_{i \beta} S_{\alpha j k}-\delta_{i \alpha} S_{\beta j k}+\delta_{i, n+\beta} S_{n+\alpha, j, k}- \\
&-\delta_{i, n+\alpha} S_{n+\beta, j, k}+\delta_{j \beta} S_{i \alpha k}-\delta_{j \alpha} S_{i \beta k}+\delta_{j, n+\beta} S_{i, n+\alpha, k}-\delta_{j, n+\alpha} S_{i, n+\beta k}+ \\
&+\delta_{k \beta} S_{i j \alpha}-\delta_{k \alpha} S_{i j \beta}+\delta_{k, n+\beta} S_{i, j, n+\alpha}-\delta_{k, n+\alpha} S_{i, j, n+\beta}=0 .
\end{aligned}
$$

Для компонент $S_{\alpha, \beta, n+\alpha}(\alpha<\beta)$ имеем

$$
-x^{n+\alpha} \partial_{n+\beta} S_{\alpha, \beta, n+\alpha}-S_{\alpha, \beta, n+\beta}=0,
$$

т.е. $-x^{n+\alpha} \cdot 1+\left(x^{n+\alpha}+c^{n+\alpha}\right)=0$, откуда $c^{n+\alpha}=0$. Для компонент $S_{\alpha, \beta, n+\beta}(\alpha<\beta)$ имеем

$$
x^{n+\beta} \partial_{n+\alpha} S_{\alpha, \beta, n+\beta}-S_{\alpha, \beta, n+\alpha}=0,
$$

т.е. $x^{n+\beta} \cdot(-1)+\left(x^{n+\beta}+c^{n+\beta}\right)=0$, откуда $c^{n+\beta}=0$.

Аналогично, выписывая уравнения инвариантности, для операторов $X_{n+\beta}^{\beta}$ находим, что $c^{\alpha}=0$ и $c^{\beta}=0$, поэтому

$$
\begin{aligned}
& S_{\alpha, \beta, n+\beta}=S_{\beta, n+\beta, \alpha}=S_{n+\beta, \alpha, \beta}=-S_{\beta, \alpha, n+\beta}=-S_{n+\beta, \beta, \alpha}=-S_{\alpha, n+\beta, \beta}=-x^{n+\alpha}, \quad \alpha<\beta, \\
& S_{\alpha, \beta, n+\alpha}=S_{\beta, n+\alpha, \alpha}=S_{n+\alpha, \alpha, \beta}=-S_{\beta, \alpha, n+\alpha}=-S_{n+\alpha, \beta, \alpha}=-S_{\alpha, n+\alpha, \beta}=x^{n+\beta}, \quad \alpha<\beta .
\end{aligned}
$$

Далее, исследуя уравнения инвариантности для операторов вращения, заключаем, что на компоненты $S_{i j k}$, содержащие хотя бы один индекс $m$, условий нет, а остальные компоненты, не содержащие индекс $m$, необходимо равны нулю. Таким образом, условия ковариантного постоянства контактной формы и инвариантность связности относительно автоморфизмов сасакиевой структуры однозначно определяют форму связности и, следовательно, связность $\widetilde{\nabla}$. Форма связности имеет следующий вид $(\alpha<\beta)$ :

$$
\begin{aligned}
S=\sum_{\alpha=1}^{n} d x^{\alpha} \wedge d x^{n+\alpha} \wedge d x^{m}+\sum_{\alpha, \beta=1}^{n} x^{n+\beta} d x^{\alpha} \wedge & d x^{\beta} \wedge d x^{n+\alpha}-\sum_{\alpha, \beta=1}^{n} x^{n+\alpha} d x^{\alpha} \wedge d x^{\beta} \wedge d x^{n+\beta}= \\
& =\left(\sum_{\alpha=1}^{n} d x^{\alpha} \wedge d x^{n+\alpha}\right) \wedge\left(d x^{m}-\sum_{\alpha=1}^{n} x^{n+\alpha} d x^{\alpha}\right),
\end{aligned}
$$

т.е. $S=d \eta \wedge \eta$. Теперь инвариантность формы кручения относительно автоморфизмов сасакиевой структуры очевидна, так как

$$
L_{X}(d \eta \wedge \eta)=L(d \eta) \wedge \eta+d \eta \wedge L_{X} \eta=d\left(L_{X} \eta\right) \wedge \eta+d \eta \wedge L_{X} \eta=0 .
$$

Таким образом, вычислительная формула для коэффициентов $\widetilde{\Gamma}_{i j}^{k}$ связности $\widetilde{\nabla}$ имеет вид

$$
\widetilde{\Gamma}_{i j}^{k}=\frac{1}{2} g^{k p}\left(\partial_{i} g_{p j}+\partial_{j} g_{i p}-\partial_{p} g_{i j}+(d \eta \wedge \eta)_{i j p}\right)
$$

а в бескоординатной форме имеем искомую формулу.

Теорема 2. Линейная связность $\widetilde{\nabla}$ является контактной метрической связностью для любой k-контактной метрической структуры.

Доказательство. Так как связность $\widetilde{\nabla}$ имеет кососимметрическое кручение, то она является метрической. Докажем, что она является и контактной для любой $k$-контактной метрической структуры. Известно (см., например, $[6,10]$ ), что на гладком многообразии существует атлас Дарбу, в каждой карте которого контактная форма $\eta$ имеет вид

$$
\eta=-x^{n+1} d x^{1}-\ldots-x^{2 n} d x^{n}+d x^{m} .
$$

В координатах Дарбу имеем

$$
d \eta=d x^{1} \wedge d x^{n}+\ldots+d x^{n} \wedge d x^{2 n}, \quad \xi=\partial_{m}, \quad \xi^{i}=g^{i s} \eta_{s}=\delta_{m}^{i}
$$


Найдем ковариантную производную от контактной формы

$$
\begin{aligned}
\widetilde{\nabla}_{i} \eta_{i}=\partial_{i} \eta_{j}-\widetilde{\Gamma}_{i j}^{k} \eta_{k}=\partial_{i} \eta_{j}-\frac{1}{2} g^{k p}\left(\partial_{i} g_{p j}+\partial_{j} g_{i p}-\partial_{p} g_{i j}\right) \eta_{k}-\frac{1}{2} S_{i j p} g^{p k} \eta_{k}= \\
=\partial_{i} \eta_{j}-\frac{1}{2}\left(\partial_{i} g_{m j}+\partial_{j} g_{i m}-\partial_{m} g_{i j}\right)-\frac{1}{2} S_{i j m}
\end{aligned}
$$

Так как $S_{i j m}=(d \eta \wedge \eta)_{i j m}$, а $\eta_{m}=1$, то $S_{i j m}=(d \eta)_{i j}$. Поскольку мы имеем $k$-контактную структуру, т.е. контактная форма $\eta$ является формой Киллинга, то вектор $\xi$ есть вектор Киллинга и, следовательно, уравнения Киллинга

$$
\xi^{k} \partial_{k} g_{i j}+\partial_{i} \xi^{k} g_{k j}+\partial_{j} \xi^{k} g_{i k}=0
$$

обращаются в тождества, откуда следует, что $\partial_{m} g_{i j}=0$. Теперь ковариантная производная от формы $\eta$ примет вид

$$
\widetilde{\nabla}_{i} \eta_{j}=\partial_{i} \eta_{j}-\frac{1}{2}\left(\partial_{i} g_{m j}+\partial_{j} g_{i m}\right)-\frac{1}{2}(d \eta)_{i j} .
$$

Далее из условия (5) определения контактной метрической структуры следует, что

$$
g(X, Y)=h(X, Y)+\eta(X) \eta(Y),
$$

где $h(X, Y)=g(\Phi X, \Phi Y)$. Структурный эндоморфизм $\Phi$ определяется условием $(d \eta)_{i j}=g_{i s} \Phi_{j}^{s}$, поэтому $\Phi_{j}^{i}=g^{i s}(d \eta)_{s j}$ и, следовательно,

$$
h_{i j}=g_{s p} \Phi_{i}^{s} \Phi_{j}^{p}=g_{s p} g^{s l}(d \eta)_{l i} g^{p k}(d \eta)_{k j} .
$$

Но $(d \eta)_{i m}=0$, тогда $h_{i m}=h_{m i}=0$ и, следовательно,

$$
g_{m j}=\eta_{m} \eta_{j}=\eta_{j}, \quad g_{i m}=\eta_{i} \eta_{m}=\eta_{i} .
$$

Поэтому

$$
\widetilde{\nabla}_{i} \eta_{j}=\partial_{i} \eta_{j}-\frac{1}{2}\left(\partial_{i} \eta_{j}+\partial_{j} \eta_{i}\right)-\frac{1}{2}\left(\partial_{i} \eta_{j}-\partial_{j} \eta_{i}\right)=\frac{1}{2} \partial_{i} \eta_{j}-\frac{1}{2} \partial_{j} \eta_{i}-\frac{1}{2} \partial_{i} \eta_{j}+\frac{1}{2} \partial_{j} \eta_{i}=0 .
$$

Так как любая сасакиева структура является $k$-контактной (см. $[6,10])$, то данная теорема верна и для любой сасакиевой структуры.

3. Субримановы геодезические. На группе Гейзенберга рассмотрим неголономное поле реперов $\left\{p, e_{i}\right\}$, адаптированное к структуре почти произведения $\mathfrak{L} \oplus \mathfrak{M}$ :

$$
e_{\alpha}=\partial_{\alpha}+x_{n+\alpha} \partial_{m}, \quad e_{n+\alpha}=\partial_{n+\alpha}, \quad e_{m}=\partial_{m},
$$

где первые $2 n$ векторных полей принадлежат контактному распределению, а $e_{m}=\xi$. Разложение коммутаторов координатных векторных полей

$$
\left[e_{i}, e_{j}\right]=\Omega_{i j}^{k} e_{k}
$$

являются структурными уравнениями поля реперов $\left\{p, e_{i}\right\}$, а коэффициенты разложения $\Omega_{i j}^{k}$ определяют объект неголономности. Для рассматриваемого поля реперов имеем

$$
\left[e_{\alpha}, e_{\beta}\right]=0, \quad\left[e_{i}, e_{m}\right]=0, \quad\left[e_{\alpha}, e_{n+\alpha}\right]=-e_{m}, \quad\left[e_{\alpha}, e_{n+\beta}\right]=0, \quad \alpha \neq \beta .
$$

Таким образом, $\Omega_{\alpha n+\alpha}^{m}=-\Omega_{n+\alpha \alpha}^{m}=-1$, остальные компоненты равны нулю. Дуальный реперу $\left\{p, e_{i}\right\}$ корепер $\left\{p, \theta^{j}\right\}$ определяется условием $\theta^{j}\left(e_{i}\right)=\delta_{i}^{j}$ и имеет вид

$$
\theta^{\alpha}=d x^{\alpha}, \quad \theta^{n+\alpha}=d x^{n+\alpha}, \quad \theta^{m}=-x^{n+1} d x^{1}-\ldots-x^{2 n} d x^{n}+d x^{m} .
$$

Левоинвариантная метрика в неголономном репере $\left\{p, e_{i}\right\}$ имеет следующий вид

$$
g(X, X)=X^{1^{2}}+X^{2^{2}}+\ldots+X^{m 2},
$$

где $X^{i}$ - неголономные координаты вектора $X=X^{i} e_{i}$, а репер $\left\{p, e_{i}\right\}$ является ортонормированным. 
Вычислим неголономные коэффициенты $\gamma_{i j}^{k}$ связности Леви-Чивиты $\nabla$ и неголономные коэффициенты $\widetilde{\gamma}_{i j}^{k}$ контактной метрической связности $\widetilde{\nabla}$ :

$$
\nabla_{e_{i}} e_{j}=\gamma_{i j}^{k} e_{k}, \quad \widetilde{\nabla}_{e_{i}} e_{j}=\widetilde{\gamma}_{i j}^{k} e_{k} .
$$

Для связности Леви-Чивиты имеем следующую вычислительную формулу (см., например, [5])

$$
g\left(\nabla_{X} Y, Z\right)=\frac{1}{2}(X g(Y, Z)+Y g(Z, X)-Z g(X, Y)+g(Z,[X, Y])+g(Y,[Z, X])-g(X,[Y, Z])) .
$$

Полагая $X=X^{i} e_{i}, Y=Y^{j} e_{j}, Z=Z^{k} e_{k}$, получим

$$
\gamma_{i j}^{k}=\frac{1}{2} \delta^{k s}\left(e_{i}\left(\delta_{s j}\right)+e_{j}\left(\delta_{i s}\right)-e_{s}\left(\delta_{i j}\right)+\Omega_{i j}^{l} \delta_{s l}+\Omega_{s i}^{l} \delta_{j l}-\Omega_{j s}^{l} \delta_{i l}\right) .
$$

Так как $e_{i}\left(\delta_{j k}\right)=0$ и $\Omega_{i j}^{k}=-\Omega_{j i}^{k}$, то

откуда находим, что

$$
\gamma_{i j}^{k}=\frac{1}{2}\left(\Omega_{i j}^{k}+\delta^{k s} \Omega_{s i}^{l} \delta_{j l}+\delta^{k s} \Omega_{s j}^{l} \delta_{i l}\right),
$$

$$
\gamma_{n+\alpha, \alpha}^{m}=\frac{1}{2}, \quad \gamma_{\alpha, n+\alpha}^{m}=-\frac{1}{2}, \quad \gamma_{\alpha m}^{n+\alpha}=\gamma_{m \alpha}^{n+\alpha}=\frac{1}{2}, \quad \gamma_{n+\alpha, m}^{\alpha}=\gamma_{m, n+\alpha}^{\alpha}=-\frac{1}{2}
$$

Так как

$$
\widetilde{\gamma}_{i j}^{k}=\gamma_{i j}^{k}+\frac{1}{2} S_{i j}^{k}
$$

где $S_{i j}^{k}=\delta^{k s} S_{i j s}$ - неголономные коэффициенты тензора кручения $\widetilde{S}$, а $S_{i j s}$ - компоненты формы кручения

$$
d \eta \wedge \eta=\theta^{1} \wedge \theta^{n+1} \wedge \theta^{m}+\ldots+\theta^{n} \wedge \theta^{2 n} \wedge \theta^{m} .
$$

Поэтому

$$
\begin{gathered}
S_{\alpha, n+\alpha, m}=S_{n+\alpha, m, \alpha}=S_{m, \alpha, n+\alpha}=-S_{n+\alpha, \alpha, m}=-S_{m, n+\alpha, \alpha}=-S_{\alpha, m, n+\alpha}=1, \\
S_{\alpha, n+\alpha}^{m}=S_{n+\alpha, m}^{\alpha}=S_{m \alpha}^{n+\alpha}=1, \quad S_{n+\alpha, \alpha}^{m}=S_{m, n+\alpha}^{\alpha}=S_{\alpha m}^{n+\alpha}=-1,
\end{gathered}
$$

остальные коэффициенты равны нулю. Теперь находим ненулевые коэффициенты связности $\widetilde{\nabla}$ :

$$
\widetilde{\gamma}_{m \alpha}^{n+\alpha}=1, \quad \widetilde{\gamma}_{m, n+\alpha}^{\alpha}=-1 \quad\left(\widetilde{\nabla}_{e_{m}} e_{\alpha}=e_{n+\alpha}, \quad \widetilde{\nabla}_{e_{m}} e_{n+\alpha}=-e_{\alpha}\right) .
$$

Контактное распределение $\mathfrak{L}=\operatorname{ker} \eta 2 n$-мерных площадок вместе с ограничением метрики $g$ на это распределение

$$
d s_{\mathfrak{L}}^{2}=d x^{1^{2}}+\ldots+d x^{2 n^{2}}
$$

определяет на группе Гейзенберга субриманову структуру. Горизонтальная (допустимая) кривая $\gamma: x^{i}=x^{i}(s)$ (s-естественный параметр) называется субримановой геодезической, если она является геодезической относительно усеченной связности $\bar{\nabla}: \bar{\nabla}_{\dot{\gamma}} \dot{\gamma}=0$, где $\bar{\nabla}$ - ортогональная проекция связности $\widetilde{\nabla}$ (или $\nabla$ ) на распределение $\mathfrak{L}$, а $\dot{\gamma}-$ поле касательных векторов кривой $\gamma$, лежащих в распределении $\mathfrak{L}$. Так как $\widetilde{\nabla}_{e_{i}} e_{j}=0$ и $\nabla_{e_{i}} e_{j}=0, i, j=\overline{1,2 n}$, то проекции контактной метрической связности $\widetilde{\nabla}$ и связности Леви-Чивиты $\nabla$ совпадают и, следовательно, определяют одну и ту же усеченную связность: $\bar{\nabla}_{e_{i}} e_{j}=0$.

Найдем сначала уравнения параллельного перенесения допустимых векторов вдоль горизонтальных кривых. Как обычно, векторное поле $v$ назовем параллельным вдоль кривой $\gamma$, если $\bar{\nabla}_{\dot{\gamma}} v=0$. Пусть $\dot{x}^{i}$ и $v^{i}$ - естественные координаты векторных полей $\dot{\gamma}$ и $v$, т.е.

$$
\dot{\gamma}=\dot{x}^{i} \partial_{i}, \quad v=v^{k} \partial_{k} \quad i, k=\overline{1, m},
$$

а $\xi^{i}$ и $\eta^{k}$-их координаты в ортонормированном базисе $e_{i}(i=\overline{1,2 n})$ контактного распределения $\mathfrak{L}$, т.е.

Так как

$$
\dot{\gamma}=\xi^{i} e_{i}, \quad v=\eta^{k} e_{k}
$$

$$
\partial_{\alpha}=e_{\alpha}-x_{n+\alpha} e_{m}, \quad \partial_{n+\alpha}=e_{n+\alpha}, \quad \partial_{m}=e_{m},
$$


TO

$$
\dot{\gamma}=\dot{x}^{i} \partial_{i}=\dot{x}^{\alpha}\left(e_{\alpha}-x_{n+\alpha} e_{m}\right)+\dot{x}^{n+\alpha} e_{n+\alpha}+\dot{x}^{m} e_{m}=\dot{x}^{i} e_{i}+\left(\dot{x}^{m}-\dot{x}^{\alpha} x_{n+\alpha}\right) e_{m} \quad(i=\overline{1,2 n}) .
$$

Поэтому $\dot{x}^{i}=\xi^{i}(i=\overline{1,2 n})$, а $\dot{x}^{m}-\dot{x}^{\alpha} x_{n+\alpha}=0$, так как $\dot{\gamma} \in \mathfrak{L}$. Аналогично,

$$
v=v^{k} \partial_{k}=v^{k} e_{k}+\left(v^{m}-v^{\alpha} x_{n+\alpha}\right) e_{m}
$$

и, следовательно, $v^{k}=\eta^{k}(k=\overline{1,2 n})$, а $v^{m}-v^{\alpha} x_{n+\alpha}=0$, так как $v \in \mathfrak{L}$. Далее имеем

$$
\bar{\nabla}_{\dot{\gamma}} v=\xi^{i} \bar{\nabla}_{e_{i}}\left(\eta^{k} e_{k}\right)=\xi^{i} e_{i}\left(\eta^{k}\right) e_{k}+\xi^{i} \eta^{k} \bar{\nabla}_{e_{i}} e_{k}=\xi^{i} e_{i}\left(\eta^{k}\right) e_{k} .
$$

Поэтому уравнения параллельного перенесения примут вид

$$
\xi^{i} e_{i}\left(\eta^{k}\right)=0
$$

Перепишем эти уравнения в естественных координатах, учитывая, что

$$
\begin{gathered}
\xi^{i}=\dot{x}^{i}, \quad \eta^{k}=v^{k} \quad(i, k=\overline{1,2 n}), \\
e_{\alpha}=\partial_{\alpha}+x_{n+\alpha} \partial_{m}, \quad e_{n+\alpha}=\partial_{n+\alpha} .
\end{gathered}
$$

В результате получим

$$
\dot{x}^{\alpha}\left(\partial_{\alpha} v^{k}+x_{n+\alpha} \partial_{m} v^{k}\right)+\dot{x}^{n+\alpha} \partial_{n+\alpha} v^{k}=0
$$

Ho

поэтому получим

$$
\dot{x}^{\alpha} x_{n+\alpha}=\dot{x}^{m}, \quad(\dot{\gamma} \in \mathfrak{L}),
$$

$$
\dot{x}^{\alpha} \partial_{\alpha} v^{k}+\dot{x}^{m} \partial_{m} v^{k}+\dot{x}^{n+\alpha} \partial_{n+\alpha} v^{k}=0,
$$

т.е. $\dot{x}^{p} \partial_{p} v^{k}=0(p=\overline{1, m} ; k=\overline{1,2 n})$ или $\dot{v}^{k}=0$. А так как $v \in \mathfrak{L}$, то $v^{m}=x^{\alpha} x_{n+\alpha}$ и, следовательно, уравнения параллельного перенесения имеют вид

$$
\dot{v}^{k}=0, \quad k=\overline{1,2 n}, \quad v^{m}=v^{\alpha} x_{n+\alpha}, \quad \alpha=\overline{1, n} .
$$

Положив теперь $v^{i}=\dot{x}^{i}$, получим дифференциальные уравнения субримановых геодезических

$$
\ddot{x}^{k}=0, \quad k=\overline{1,2 n}, \quad \dot{x}^{m}=\dot{x}^{\alpha} x_{n+\alpha}, \quad \alpha=\overline{1, n} .
$$

Интегрируя полученные уравнения, находим общее решение

$$
x^{k}=a^{k} s+b^{k}, \quad x^{m}=\frac{1}{2}\left(a_{\alpha} a^{n+\alpha}\right) s^{2}+\left(a_{\alpha} b^{n+\alpha}\right) s+b^{m}, \quad a_{\alpha}=a^{\alpha} .
$$

множество всех субримановых геодезических на группе Гейзенберга.

При исследовании строения геодезических, в силу левоинвариантности субримановой структуры, можно ограничиться геодезическими, выходящими из единицы группы

$$
x^{k}=a^{k} s, \quad x^{m}=\frac{1}{2}\left(a^{1} a^{n+1}+\ldots+a^{n} a^{2 n}\right) s^{2} .
$$

Заметим, что ортогональные проекции геодезических на контактную плоскость являются прямыми линиями. Чтобы провести анализ строения геодезических, отождествим матрицы группы Гейзенберга с точками $m$-мерного числового пространства $\mathbb{R}^{m}$, поставив в соответствие каждой матрице группы $G$ с определяющими элементами $x^{i}$ точку $x=\left(x^{i}\right)$ из $\mathbb{R}^{m}$. Так как в единице группы

$$
e_{\alpha}=\partial_{\alpha}, \quad e_{n+\alpha}=\partial_{n+\alpha}, \quad e_{m}=\partial_{m}
$$

то ортонормированному реперу $\left\{e, e_{i}\right\}$ группы $G$ будет отвечать репер $\left\{O, e_{i}\right\}$ в $\mathbb{R}^{m}$, где $O=$ $(0, \ldots, 0), e_{1}=(1,0, \ldots, 0), \ldots, e_{m}=(0, \ldots, 0,1)$. Этот репер является ортонормированным относительно стандартной евклидовой метрики $\rho_{E}$ в $\mathbb{R}^{m}$. Контактной плоскости в единице группы соответствует координатная плоскость векторов $e_{1}, \ldots, e_{2 n}$. Теперь мы можем исследовать строение субримановых геодезических, используя геометрию евклидова пространства $\mathbb{E}^{m}=\left(\mathbb{R}^{m}, \rho_{E}\right)$.

Рассмотрим несколько подробнее трехмерный случай. В евклидовом пространстве параметрические уравнения субримановых геодезических примут вид

$$
x=a_{1} s, \quad y=a_{2} s, \quad z=\frac{1}{2} a_{1} a_{2} s^{2},
$$


где $x=x^{1}, y=x^{2}, z=x^{3}, a_{i}=a^{i}$. Так как $s$ - естественный параметр, то $a_{1}^{2}+a_{2}^{2}=1$, и единичный вектор $a\left(a_{1}, a_{2}\right)$ в контактной плоскости $x O y$ определяет направление геодезической, т.е. он является касательным вектором к геодезической в начале координат. В координатной плоскости $x O y$ рассмотрим числовую прямую $l$, заданную первыми двумя уравнениями геодезических с единичным вектором $a$ и параметром $s$. Обозначим направленный угол между единичным вектором $e_{1}$ оси $O x$ и вектором $a$ через $\theta=\left(\widehat{e_{1}, a}\right)$. Тогда уравнения геодезических примут вид:

$$
x=s \cos \theta, \quad y=s \sin \theta, \quad z=\frac{1}{2} s^{2} \cos \theta \sin \theta .
$$

Первые два уравнения в плоскости $x O y$ определяют прямую $l$, а в пространстве - плоскость $l O z$. Третье уравнение в плоскости $l O z$ определяет параболу, а в пространстве - параболический цилиндр. Искомая система определяет субриманову геодезическую как пересечение параболического цилиндра и плоскости. Это пересечение является параболой, если $\cos \theta \sin \theta \neq 0$ (ее уравнение в репере $\left\{O, a, e_{3}\right\}$ является третьим уравнением системы). Если $\cos \theta \sin \theta=0$, то при $\sin \theta=0$ $(z=0)$, геодезической является ось $O x=(x O y) \cap(x O z)$. Если $\cos \theta=0$, то геодезической является ось $O y=(x O y) \cap(y O z)$. При малых $\theta$ имеем $\sin \theta \rightarrow 0$, коэффициент $\cos \theta \sin \theta>0$ мал, ветви параболы расположены в полупространстве $z>0$, и парабола мало отличается от прямой $O x$. $\mathrm{C}$ возрастанием $\theta$ это отличие растет, и при $\theta=\pi / 4$ достигает своего максимума. При $\theta>\pi / 4$ происходит «распрямление» параболы, и при $\theta=\pi / 2, \cos \theta=0$, парабола «превращается» в прямую Oy. При $\theta>\pi / 2$ ветви параболы опускаются «вниз» $(\cos \theta \sin \theta<0, \pi / 2<\theta<\pi)$. С увеличением $\theta$ парабола становится круче, достигает максимума при $\theta=3 \pi / 4$, затем опять распрямляется и становится прямой $O x$ при $\theta=\pi$. Далее ветви параболы опять поднимутся вверх. В третьем координатном угле $(\pi<\theta<3 \pi / 2)$ имеем те же параболы, что и в первом $(0<\theta<\pi / 2)$, а в четвертом $(3 \pi / 2<\theta<2 \pi)$ имеем то же самое, что и во втором $(\pi / 2<\theta<\pi)$, т.е. направления $a$ и $-a$ определяют одну и ту же геодезическую (система обладает свойством симметрии). Напомним, что в каждой точке геодезической касательный к ней вектор лежит в соответствующей контактной плоскости.

Аналогичное поведение геодезических мы наблюдаем и в многомерном случае. Если допустимые направления $а$ лежат в координатных плоскостях $\left(e_{\alpha}, e_{n+\alpha}\right)$, то геодезические ведут себя так же, как и в трехмерном случае. Из уравнений геодезических следует, что все координатные прямые репера $\left\{O, e_{i}\right\}$ являются геодезическими, кроме того, все прямые, лежащие в координатных плоскостях, отличных от $\left(e_{\alpha}, e_{n+\alpha}\right)$, также являются геодезическими.

4. Секционная кривизна. Имея коэффициенты связности $\widetilde{\nabla}$, нетрудно вычислить компоненты $\widetilde{R}_{i j k}^{l}$ тензора кривизны

$$
\begin{gathered}
\widetilde{R}(X, Y) Z=\widetilde{\nabla}_{X} \widetilde{\nabla}_{Y} Z-\widetilde{\nabla}_{Y} \widetilde{\nabla}_{X} Z-\widetilde{\nabla}_{[X, Y]} Z \\
\widetilde{R}_{i j k}^{l}=e_{i}\left(\widetilde{\gamma}_{j k}^{l}\right)-e_{j}\left(\widetilde{\gamma}_{i k}^{l}\right)+\widetilde{\gamma}_{i s}^{l} \widetilde{\gamma}_{j k}^{s}-\widetilde{\gamma}_{j s}^{l} \widetilde{\gamma}_{i k}^{s}-\Omega_{i j}^{s} \widetilde{\gamma}_{s k}^{l} .
\end{gathered}
$$

В результате получаем следующие ненулевые компоненты тензора кривизны:

$$
\begin{gathered}
\widetilde{R}_{\alpha, n+\alpha, \alpha}^{n+\alpha}=-\widetilde{R}_{\alpha, n+\alpha, n+\alpha}^{\alpha}=1, \quad \widetilde{R}_{n+\alpha, \alpha, \alpha}^{n+\alpha}=-\widetilde{R}_{n+\alpha, \alpha, n+\alpha}^{\alpha}=-1, \\
\widetilde{R}_{\alpha, n+\alpha, \alpha, n+\alpha}=-\widetilde{R}_{n+\alpha, \alpha, \alpha, n+\alpha}=-\widetilde{R}_{\alpha, n+\alpha, n+\alpha, \alpha}=\widetilde{R}_{n+\alpha, \alpha, n+\alpha, \alpha}=1 .
\end{gathered}
$$

Прямыми вычислениями нетрудно убедиться, что в связности $\widetilde{\nabla}$ тензоры кручения и кривизны ковариантно постоянны:

$$
\widetilde{\nabla} \widetilde{S}=0, \quad \widetilde{\nabla} \widetilde{R}=0 .
$$

Действительно, для ковариантных компонент тензора кручения имеем

$$
\widetilde{\nabla}_{l} S_{i j k}=e_{l}\left(S_{i j k}\right)-S_{p j k} \widetilde{\gamma}_{l i}^{p}-S_{i p k} \widetilde{\gamma}_{l j}^{p}-S_{i j p} \widetilde{\gamma}_{l k}^{p}
$$

Но $e_{l}\left(S_{i j k}\right)=0$, а для ненулевых компонент при $i=\alpha, j=n+\alpha, k=m$ имеем

$$
-\widetilde{\nabla}_{e_{l}} S_{\alpha, n+\alpha, m}=S_{\alpha, n+\alpha, m} \widetilde{\gamma}_{l \alpha}^{\alpha}+S_{\alpha, n+\alpha, m} \widetilde{\gamma}_{l, n+\alpha}^{n+\alpha}+S_{\alpha, n+\alpha, m} \widetilde{\gamma}_{l m}^{m} \equiv 0,
$$


так как $\widetilde{\gamma}_{l \alpha}^{\alpha}=\widetilde{\gamma}_{l, n+\alpha}^{n+\alpha}=\widetilde{\gamma}_{l m}^{m}=0$. Аналогично для тензора кривизны:

$$
\begin{gathered}
\widetilde{\nabla}_{e_{r}} \widetilde{R}_{i j k l}=e_{r}\left(\widetilde{R}_{i j k l}\right)-\widetilde{R}_{p j k l} \widetilde{\gamma}_{r i}^{p}-\widetilde{R}_{i p k l} \widetilde{\gamma}_{r j}^{p}-\widetilde{R}_{i j p l} \widetilde{\gamma}_{r k}^{p}-\widetilde{R}_{i j k p} \widetilde{\gamma}_{r l}^{p}, \\
-\widetilde{\nabla}_{e_{r}} \widetilde{R}_{\alpha, n+\alpha, \alpha, n+\alpha}=\widetilde{R}_{\alpha, n+\alpha, \alpha, n+\alpha} \widetilde{\gamma}_{r \alpha}^{\alpha}+\widetilde{R}_{\alpha, n+\alpha, \alpha, n+\alpha} \widetilde{\gamma}_{r, n+\alpha}^{n+\alpha}+\widetilde{R}_{\alpha, n+\alpha, \alpha, n+\alpha} \widetilde{\gamma}_{r, n+\alpha}^{n+\alpha} \equiv 0 .
\end{gathered}
$$

Заметим, что ковариантный тензор кривизны $g(\widetilde{R}(X, Y) Z, W)$ кососимметричен по первой и второй паре аргументов, и эти пары можно менять местами. Поэтому мы можем ввести понятие секционной кривизны аналогично тому, как это делается в римановой геометрии, т.е. в каждой точке $p \in G$ и в каждом двумерном направлении $\mathfrak{S}$, определяемом векторами $u, v \in T_{p} G$, кривизна $\widetilde{k}$ определяется формулой

$$
\widetilde{k}=\frac{g(\widetilde{R}(u, v) v, u)}{g(u, u) \cdot g(v, v)-g(u, v)^{2}}
$$

Теорема 3. Секционная кривизна $\widetilde{k}$ группь Гейзенберга с контактной метрической связностъю принадлежит числовому отрезку $[-1,0]$.

Доказательство. Будем считать, что векторы $u, v \in \mathfrak{S}_{p}$ единичны и ортогональны, тогда

$$
\begin{array}{r}
\widetilde{k}=g(\widetilde{R}(u, v) v, u)=\widetilde{R}_{\alpha, n+\alpha, \alpha, n+\alpha} u^{\alpha} v^{n+\alpha} v^{\alpha} u^{n+\alpha}+\widetilde{R}_{n+\alpha, \alpha, \alpha, n+\alpha} u^{n+\alpha} v^{\alpha} v^{\alpha} u^{n+\alpha}+ \\
+\widetilde{R}_{\alpha, n+\alpha, n+\alpha, \alpha} u^{\alpha} v^{n+\alpha} v^{n+\alpha} u^{\alpha}+\widetilde{R}_{n+\alpha, \alpha, n+\alpha, \alpha} u^{n+\alpha} v^{\alpha} v^{n+\alpha} u^{\alpha}= \\
=-\left[\left(\left(u^{1}\right)^{2}\left(v^{n+1}\right)^{2}+\ldots+\left(u^{n}\right)^{2}\left(v^{2 n}\right)^{2}\right)-2\left(u^{1} v^{1} u^{n+1} v^{n+1}+\ldots+u^{n} v^{n} u^{2 n} v^{2 n}\right)+\right. \\
\left.+\left(\left(u^{n+1}\right)^{2}\left(v^{1}\right)^{2}+\ldots+\left(u^{2 n}\right)^{2}\left(v^{n}\right)^{2}\right)\right] .
\end{array}
$$

Пусть $m=3$; тогда

$$
\widetilde{k}=-\left(u^{1} v^{2}-u^{2} v^{1}\right)^{2} \leqslant 0 .
$$

Если поле двумерных направлений $\mathfrak{S}$ определяется контактным распределением $\mathfrak{L}$, то в качестве вектора $v$ можно взять $\Phi u$, где $\Phi$-структурный эндоморфизм, действующий в $\mathfrak{L}$ как оператор комплексной структуры, т.е. $v^{1}=-u^{2}, v^{2}=u^{1}$. Тогда

$$
\widetilde{k}=-\left(\left(u^{1}\right)^{2}+\left(u^{2}\right)^{2}\right)=-1
$$

так как вектор $u$ - единичный. Если плоскость $\mathfrak{S}_{p}$ содержит вектор $\xi(0,0,1)$, то $\widetilde{k}=0$. Если плоскость $\mathfrak{S}_{p}$ не совпадает с $\mathfrak{L}$ и не содержит вектор $\xi$, то поступая так же, как в [7], вектор $u$ возьмем в пересечении $\mathfrak{S}_{p} \cap \mathfrak{L}_{p}$, а $v$ ему перпендикулярно. Тогда $u^{1} v^{1}+u^{2} v^{2}=0$. Возводя это равенство в квадрат, получим

$$
-2 u^{1} v^{1} u^{2} v^{2}=\left(u^{1}\right)^{2}\left(v^{1}\right)^{2}+\left(u^{2}\right)^{2}\left(v^{2}\right)^{2},
$$

поэтому

$$
\widetilde{k}=-\left(\left(u^{1}\right)^{2}\left(v^{2}\right)^{2}-2 u^{1} v^{1} u^{2} v^{2}+\left(u^{2}\right)^{2}\left(v^{1}\right)^{2}\right)=-\left(\left(u^{1}\right)^{2}+\left(u^{2}\right)^{2}\right)\left(\left(v^{1}\right)^{2}+\left(v^{2}\right)^{2}\right) .
$$

Так как $\left(u^{1}\right)^{2}+\left(u^{2}\right)^{2}=1,\left(v^{1}\right)^{2}+\left(v^{2}\right)^{2}+\left(v^{3}\right)^{2}=1$, то

$$
\widetilde{k}=-\left(1-\left(u^{3}\right)^{2}\right)>-1 \text {. }
$$

Таким образом, $-1 \leqslant \widetilde{k} \leqslant 0$. Данная оценка кривизны получена в [7] с использованием естественных координат.

Пусть $m>3$; тогда, перегруппировав слагаемые, $\widetilde{k}$ можно представить так

$$
\widetilde{k}=-\left[\left(u^{1} v^{n+1}-u^{n+1} v^{1}\right)^{2}+\ldots+\left(u^{n} v^{2 n}-v^{2 n} v^{n}\right)^{2}\right] \leqslant 0 .
$$

Докажем, что $\widetilde{k} \geqslant-1$, т.е. величина в квадратных скобках $\leqslant 1$. Так же, как и в случае $m=3$, вектор $u$ возьмем в пересечении $\mathfrak{S}_{p} \cap \mathfrak{L}_{p}$. Так как $u$ и $v$ единичны и ортогональны, знаменатель в исходной формуле для $\widetilde{k}$ равен единице, однако в числителе этот факт пока не отражен. Поэтому 
векторы $u$ и $v$ мы пронормируем, разделив их координаты на длины векторов $u$ и $v$. В результате необходимо доказать, что выполняется следующее неравенство:

$$
\begin{aligned}
& {\left[\left(u^{1}\right)^{2}\left(v^{n+1}\right)^{2}+\ldots+\left(u^{n}\right)^{2}\left(u^{2 n}\right)^{2}-2 u^{1} v^{1} u^{n+1} v^{n+1}-\ldots-\right.} \\
& \left.\quad-2 u^{n} v^{n} u^{2 n} v^{2 n}+\left(u^{n+1}\right)^{2}\left(v^{1}\right)^{2}+\ldots+\left(u^{2 n}\right)^{2}\left(v^{n}\right)^{2}\right] \leqslant \\
& \leqslant\left(\left(u^{1}\right)^{2}+\ldots+\left(u^{n}\right)^{2}+\left(u^{n+1}\right)^{2}+\ldots+\left(u^{2 n}\right)^{2}\right)\left(\left(v^{1}\right)^{2}+\ldots\left(v^{n}\right)^{2}+\left(v^{n+1}\right)^{2}+\ldots+\left(v^{m}\right)^{2}\right) .
\end{aligned}
$$

Действительно, если перенести левую часть вправо, сократить слагаемые вида $\pm\left(u^{\alpha}\right)^{2}\left(v^{n+\alpha}\right)^{2}$ и $\pm\left(u^{n+\alpha}\right)^{2} v^{n 2}$ и свернуть выражения вида $\left(u^{\alpha}\right)^{2}\left(v^{\alpha}\right)^{2}+2 u^{\alpha} v^{\alpha} u^{n+\alpha} v^{n+\alpha}+\left(u^{n+\alpha}\right)^{2}\left(v^{n+\alpha}\right)^{2}$, то получим сумму квадратов вида

$$
\left(u^{1} v^{1}+u^{n+1} v^{n+1}\right)^{2}+\ldots+\left(u^{n} v^{n}+u^{2 n} v^{2 n}\right)^{2}+\left(u^{1}\right)^{2}\left(v^{2}\right)^{2}+\ldots+\left(u^{2 n}\right)^{2}\left(v^{m}\right)^{2} \geqslant 0
$$

что и доказывает наше утверждение. Таким образом, как и в случае $m=3$, для секционной кривизны выполняется неравенство $-1 \leqslant \widetilde{k} \leqslant 0$, причем, например, -1 достигается в направлениях, определяемых координатными плоскостями, натянутыми на векторы $e_{\alpha}, e_{n+\alpha}$, а 0 - оставшимися координатными плоскостями, а также в случае, когда направление $\mathfrak{S}$ содержит характеристический вектор $\xi$.

\section{СПИСОК ЛИТЕРАТУРЫ}

1. Аграчев A. А. Некоторые вопросы субримановой геометрии// Усп. мат. наук. - 2016. - 71, № 6 (432). - C. 3-36.

2. Вершик A. М., Герикович В. Я. Неголономные динамические системы. Геометрия распределений и вариационные задачи / Итоги науки и техн. Совр. пробл. мат. Фундам. напр. - 1987. - 16. - C. 5-85.

3. Вершик А. М., Фадеев Л. Д. Лагранжева механика в инвариантном изложении// в кн.: Проблемы теоретической физики. - Л.: Изд-во ЛГУ, 1975. - С. 129-141.

4. Горбацевич В. В. Субримановы геометрии на компактных однородных пространствах// Изв. РАН. Cер. мат. - 2014. - 78, № 3. - С. 35-52.

5. Громол Д., Клингенберг В., Майер В. Риманова геометрия в целом. - М.: Мир, 1971.

6. Кириченко В. Ф. Дифференциально-геометрические структуры на многообразиях. - Одесса: Печатный Дом, 2013.

7. Панъженский В. И., Климова Т. Р. Контактная метрическая связность на группе Гейзенберга// Изв. вузов. Мат. - 2018. - 11. - С. 51-59.

8. Сачков Ю. Л. Теория управления на группах Ли// Совр. мат. Фундам. напр. - 2007. - 26. - С. 5-59.

9. Agrachev A., Barilari D., Boscain U. Introduction to Riemannian and sub-Riemannian geometry. — Trieste: SISSA, 2012.

10. Blair D. E. Contact Manifolds in Riemannian geometry. — Berlin-New York: Springer-Verlag, 1976.

11. Boyer C. P. The Sasakian Geometry of the Heisenberg Group. - Albuquerque, 2009.

12. Tanno $S$. The automorphism groups of almost contact Riemannian manifolds// Tôhoku Math. J. — 1969. - 21, № 2. - P. 21-38.

Паньженский Владимир Иванович

Пензенский государственный университет

E-mail: kaf-geom@yandex.ru

Сурина Ольга Петровна

Пензенский государственный университет

E-mail: o.surina2013@yandex.ru 